\title{
A Galaxy Merging Sequence Traced by $\mathrm{X}$-rays
}

\author{
Yu Gao, Q. Daniel Wang, Ted A. Markowsky \\ Dept. of Astronomy, Univ. of Massachusetts, Amherst, MA 01003, USA
}

\begin{abstract}
We are studying a sample of nearly 20 nearby $(\mathrm{cz} \lesssim 13,000 \mathrm{~km}$ $\mathrm{s}^{-1}$ ) IR-luminous interacting/merging galaxies observed with the ACIS in the Chandra archive (e.g., Gao et al. 2003; Ptak et al. 2003). Most galaxies in the sample are luminous infrared galaxies (LIGs) and nearly half of them are ultraluminous infrared galaxies (ULIGs) with bolometric luminosities comparable to QSOs. Based on multiwavelength data and numerical simulations of gas-rich galaxy mergers, we have attempted to arrange them in a merger sequence perspective and compared their broadband X-ray emission here with the optical images (mostly DSS).
\end{abstract}

We tentatively conclude the following:

1. Extended diffuse soft $\mathrm{X}$-ray emission over $\gtrsim 100 \mathrm{kpc}$ scale was detected in some LIGs/ULIGs (e.g., Mrk 273 \& NGC 6240) of intermediate/advanced stage experiencing the merger-induced peak nuclear activities (extreme starbursts and AGN), but not in the early-stage or very late-stage mergers. In fact, most ULIGs don't show the spectacular large-scale X-ray envelopes as seen in ULIG Mrk 273. We present the broad-band X-ray images (contours) in Figure 1 as compared with the optical morphology.

2. Besides Arp 220, the nearest archetypal ULIG, all other ULIGs (from Mrk 273 to IRAS 17208-0014 in Figure 1) are at about the same distance with comparable IR luminosities, yet the X-ray properties (with similar Chandra exposure) are rather drastically different among them. Although ULIGs (NGC 6240 \& Arp 299 are also luminous enough to be nearly qualified to be ULIGs) appear to be in the same advance merging stage from their optical appearance with the apparently peak IR luminosity, the diffuse X-ray morphology might indicate the subtle difference in their evolutionary path into the giant elliptical galaxies.

3. Specifically, whether there appears to be a development of the X-ray superwind, from active on-going merging phase (nearly ULIGs) to the peak activity (ultraluminous extreme starbursts), then to a decrease of such activity, and to later further detachment of such X-ray winds from the late-stage merger remnants should be carefully examined. There might be such excess X-ray emission in the surroundings of some late-stage ULIGs.

4. A combination of the diffuse soft X-ray large-scale morphology and the hard X-ray emission in the inner disks (nuclear regions) might serve as a pivotal tool to probe the merging stages, the nuclear activities, and associated phenomena such as superwinds. Combined with multiwavelength studies and numerical simulations, better characterization of X-ray emission should provide 
the essential links all the way from normal galaxies (pre-merging), starbursts (ongoing merging), and AGN/super-starbursts (late mergers), to giant ellipticals.

\section{References}

Gao, Y., Wang, Q.D., Appleton, P.N., Lucas, R.A. 2003, ApJ, 596, L171

Ptak, A., Heckman, T., Levenson, N.A. et al. 2003, ApJ, 592, 782

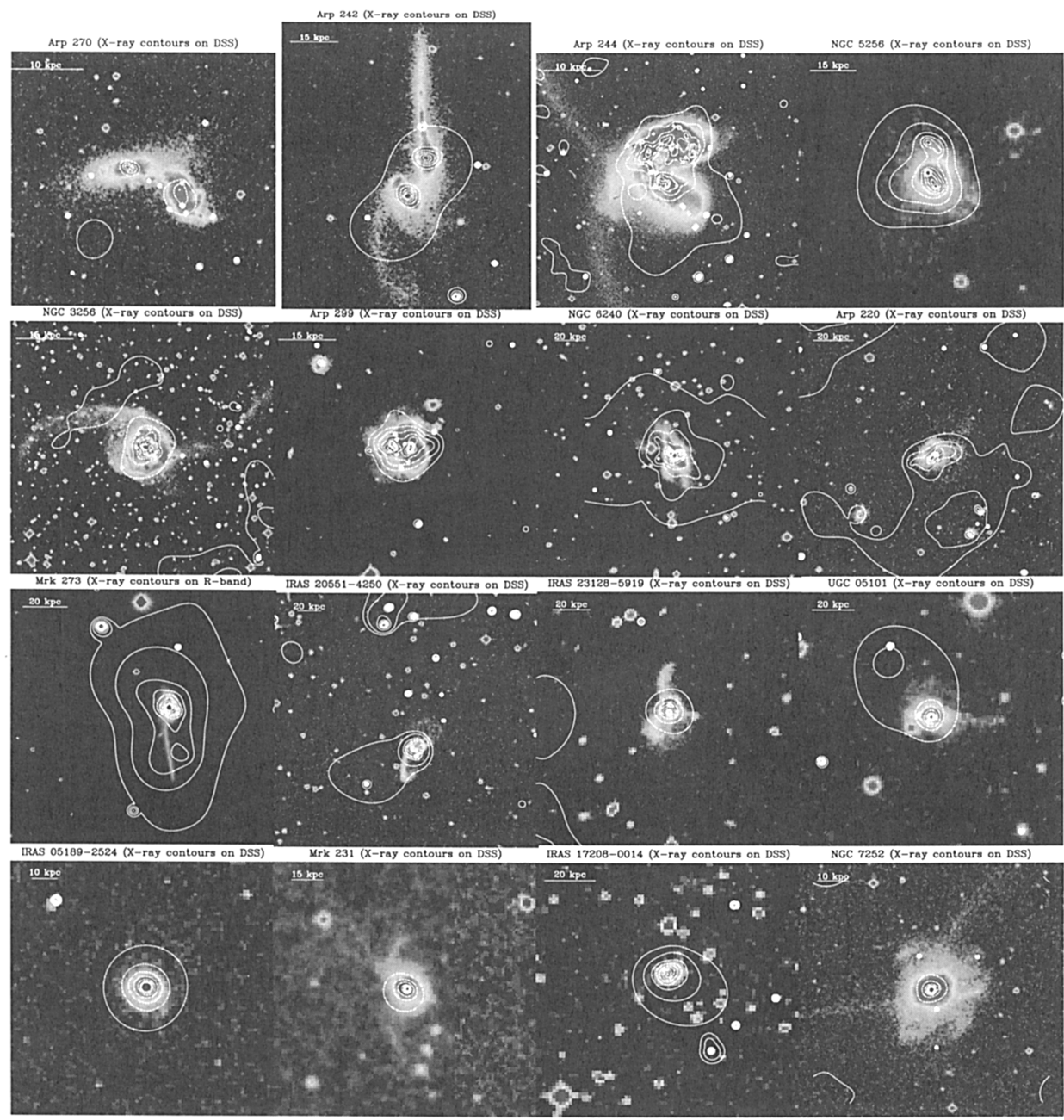

Figure 1. The broad-band X-ray images (contours) overlaid on optical images of 16 LIGs/ULIGs to illustrate the merger sequence. Contours start at $\sim 3 \sigma$ above the background and increase successively by a factor of 2 . 